\title{
FACTORIZATION OF DIFFUSIONS ON FIBRE BUNDLES
}

\author{
MING LIAO
}

\begin{abstract}
Let $\pi: M \rightarrow N$ be a fibre bundle with a $G$-structure and a connection. A $G$-invariant operator $A$ on the standard fibre $F$ is "shifted" to an operator $A^{*}$ on $M$ and a semielliptic operator $B$ on $N$ is "lifted" to an operator $\widetilde{B}$ on $M$. Let $X_{t}$ be an $A$-diffusion on $F$, let $Y_{t}$ be a $B$-diffusion on $N$ which is independent of $X_{t}$ and let $\Psi_{t}$ be its horizontal lift in the associated principal bundle. Then $Z_{t}=\Psi_{t}\left(X_{t}\right)$ is a diffusion on $M$ with generator $A^{*}+\widetilde{B}$. Conversely, such a factorization is possible only if the fibre bundle has a proper $G$-structure. In the case of a Riemannian submersion, $X, Y$ and $Z$ can be taken to be Brownian motions and the existence of a $G$-structure then means that the fibres are totally geodesic.
\end{abstract}

\section{INTRODUCTION}

Let $\pi: M \rightarrow N$ be a Riemannian submersion with totally geodesic fibres. Hermann in $[\mathrm{H}]$ proved that it is a fibre bundle with structure group the group of isometries of the standard fibre $F$. The collection of horizontal subspaces on $M$ defines a connection. Let $\bar{\pi}: G(M) \rightarrow N$ be the associated principal bundle. Each $\psi \in G(M)$ is an isometry from $F$ onto some fibre in $M$. Elworthy and Kendall proved in [EK] the following result. If $Z_{t}$ is a Brownian motion on $M$, then $Y_{t}=\pi\left(Z_{t}\right)$ is a Brownian motion on $N$. Let $\Psi_{t}$ be the horizontal lift in $G(M)$ of $Y_{t}$, then $X_{t}=\Psi_{t}^{-1}\left(Z_{t}\right)$ is a Brownian motion on $F$. Moreover, $X$ and $Y$ are independent and $Z_{t}=\Psi_{t}\left(X_{t}\right)$. They then applied this result to obtain an interesting factorization of harmonic maps.

Elworthy and Kendall's result is the motivation for the present investigation. In this paper, we will consider a general factorization on a fibre bundle with a $G$-structure and a connection. It seems that the above result is understood better under this general setting. We will also obtain a sort of converse of this factorization, i.e. if such a factorization is possible, then the fibre bundle must have a proper $G$-structure. In the case of a Riemannian submersion, and when $X, Y$ and $Z$ are Brownian motions, this means that the fibres are totally geodesic.

Received by the editors December 3, 1987.

1980 Mathematics Subject Classification (1985 Revision). Primary 58G32; Secondary 53C05.

Key words and phrases. Diffusions, elliptic operators, fibre bundles, structure groups, connections, Riemannian submersions, totally geodesic fibres.

Research supported in part by the Natural Science Foundation of PR China and NSF Grant 8318204. 
The paper is organized as follows. $\S \S 2,3$ and 4 deal with the differential geometric preparation. Let $\pi: M \rightarrow N$ be a fibre bundle with a distribution $H$ of subspaces transversal to fibres. A semielliptic operator $B$ on $N$ is lifted to an operator $\widetilde{B}$ on $M$ via $H$. With a $G$-structure, a $G$-invariant operator $A$ on the standard fibre $F$ induces an operator $A^{*}$ on $M . A^{*}$ commutes with $\widetilde{B} . A^{*}+$ $\widetilde{B}$ is nondegenerate if and only if both $A$ and $B$ are nondegenerate. In the case of a Riemannian submersion with totally geodesic fibres, $A^{*}+\widetilde{B}$ is the Laplacian on $M$ if and only if $A$ and $B$ are, respectively, the Laplacians on $F$ and $N$. Although, some of these results are not needed in the subsequent sections, they are included because they seem to be interesting in their own rights. $\S 5$ recalls the definition of diffusions on manifolds and defines the horizontal lift of a diffusion on the base manifold $N . \S \S 6$ and 7 contain the main results of this paper. In $\S 6$, we show that if $X_{t}$ is a diffusion on $F$ with a $G$-invariant generator $A, Y_{t}$ is a diffusion on $N$, which is independent of $X$ and has generator $B$, and $\Psi_{t}$ is the horizontal lift in $G(M)$ of $Y_{t}$, then $\Psi_{t}\left(X_{t}\right)$ is a diffusion on $M$ with generator $A^{*}+\widetilde{B}$. On the other hand, if $Z_{t}$ is a diffusion on $M$ and it projects down to $Y_{t}$, then $X_{t}=\Psi_{t}^{-1}\left(Z_{t}\right)$ is a diffusion on $F$ which is independent of $Y$. The factorization of Elworthy and Kendall is obtained as a corollary. In $\S 7$, we show that such a factorization is possible on a Riemannian submersion if and only if the fibres are totally geodesic. We also prove a similar result for general fibre bundles. The Appendix discusses a slight generalization of our results. This was observed by the referee.

The author wishes to thank P. Baxendale for a helpful conservation.

\section{FIBRE BUNDLES AND HORIZONTAL LIFTS}

Throughout this paper, manifolds, maps and functions will always be assumed to be smooth.

Let $N$ and $M$ be, respectively, $n$-dimensional and $(n+k)$-dimensional manifolds. An onto map $\pi: M \rightarrow N$ is said to be a fibre bundle with standard fibre $F$, a $k$-dimensional manifold, if there is an open cover $\{0\}$ of $N$ and for each 0 , there is a diffeomorphism $\phi: 0 \times F \rightarrow \pi^{-1}(0)$ such that $\pi \circ \phi$ is the projection $0 \times F \rightarrow 0 . \quad F_{y}=\pi^{-1}(y)$ is called the fibre over $y$ and is necessarily diffeomorphic to $F$.

For $z \in M$, an $n$-dimensional subspace $H_{z}$ of $T_{z} M$ will be called a transversal subspace at $z$ if $D \pi\left(H_{z}\right)=T_{y} N$ with $y=\pi(z)$, where $D \pi$ is the differential map of $\pi: M \rightarrow N$. If $H_{z}$ is a transversal subspace at $z$, we will also use the symbol $H_{z}$ for the unique linear map $H_{z}: T_{y} N \rightarrow T_{z} M$ such that $H_{z}\left(T_{y} N\right)=H_{z}$. For typographical convenience, we sometimes write $H(z)$ for $H_{z}$ and $H(z) U$ for $H_{z} U$ when $U \in T_{y} N$.

A differential operator $B$ on $N$ will be called semielliptic if for any $y \in N$, there is a neighborhood of $y$ in which

$$
B=\frac{1}{2} \sum_{i=1}^{s} U_{i} U_{i}+U^{\prime}
$$


for some smooth vector fields $U_{1}, U_{2}, \ldots, U_{s}$ and $U^{\prime}$ in that neighborhood. The choice of the vector fields and the integer $s$ is not unique. Under local coordinates $y^{1}, y^{2}, \ldots, y^{n}, B$ has the form

$$
B=(1 / 2) g^{j k}(y)\left(\partial / \partial y^{j}\right)\left(\partial / \partial y^{k}\right)+b^{j}(y)\left(\partial / \partial y^{j}\right),
$$

where $g^{j k}$ is a nonnegative definition symmetric $n \times n$ matrix. We have used the convention to omit the summation sign over repeated indices and will continue to do so. Indeed, if $U_{i}=\sigma_{i}^{j}\left(\partial / \partial y^{j}\right)$, then $g^{j k}=\sigma_{i}^{j} \sigma_{i}^{k}$. We will say that $B$ is elliptic or nondegenerate if $g^{j k}$ is strictly positive definite.

Remark 1 . If we only assume (2), $B$ is not necessarily a semielliptic operator according to our definition. However, if (2) holds with strictly positive definite $g^{j k}$, then locally $B$ can be expressed by (1) with $s=n$. Hence, $B$ is a semielliptic, in fact, an elliptic operator.

Let $H=\left\{H_{z} ; z \in M\right\}$ be a smooth distribution of transversal subspaces on $M$. For any vector field $U$ on $N, H U$ is a vector field on $M$ and is called the horizontal lift of $U$ with respect to $H$.

Let $B$ be a semielliptic operator on $N$ which is expressed locally by (1). We can define a semielliptic operator $\widetilde{B}$ on $M$ by

$$
\widetilde{B}=(1 / 2)\left(H U_{i}\right)\left(H U_{i}\right)+\left(H U^{\prime}\right) .
$$

A direct computation using local coordinates shows that this definition of $\widetilde{B}$ depends only on $B$ and $H$ and is independent of the choice of $U_{i}$ and $U^{\prime}$. Although (3) defines $\widetilde{B}$ locally, it is globally defined due to this independence property. $\widetilde{B}$ will be called the horizontal lift of $B$.

Let $G$ be a finite dimensional Lie group of diffeomorphisms $F \rightarrow F$ and for each $y \in N$, let $G_{y}$ be a collection of diffeomorphisms $\psi: F \rightarrow F_{y}$ such that $G_{y}=\{\psi \circ g ; g \in G\}$ for any $\psi \in G_{y}$. The totality of $G_{y}, y \in N$, is called a $G$-structure on the fibre bundle $\pi: M \rightarrow N$ if the local trivialization map $\phi: 0 \times F \rightarrow \pi^{-1}(0)$ can be chosen so that for any $y \in 0, \phi(y, \cdot) \in G_{y}$. In this case, $G$ is called a structure group of the fibre bundle. We will say that $\pi: M \rightarrow N$ is a $(G, F)$-bundle if it is a fibre bundle with structure group $G$ and standard fibre $F$.

Assume that $\pi: M \rightarrow N$ is a $(G, F)$-bundle. Let $G(M)$ be the union of all $G_{y}, y \in N$. Define $\bar{\pi}: G(M) \rightarrow N$ by setting $\bar{\pi}(\psi)=y$ if the image of $\psi$ is $F_{y}$. Then $\bar{\pi}: G(M) \rightarrow N$ is a principal bundle with structure group $G$ and $\pi: M \rightarrow N$ is its associated fibre bundle via the action of $G$ on $F$. See [KN].

A smooth distribution $\bar{H}=\left\{\bar{H}_{\psi} ; \psi \in G(M)\right\}$ of transversal subspaces on $G(M)$ is said to be a connection on the principal bundle $\bar{\pi}: G(M) \rightarrow N$ if for any $g \in G$ and $\psi \in G(M), D g\left(\bar{H}_{\psi}\right)=\bar{H}_{\psi g}$, where $D g$, is the differential map of $g: G(M) \rightarrow G(M)$. Fix $\psi \in G(M)$ with $y=\bar{\pi}(\psi)$. Any curve $y_{t}$ in $N$ with $y_{0}=y$ is uniquely lifted to a curve $\psi_{t}$ in $G(M)$ with $\psi_{0}=\psi$ such that $\bar{\pi}\left(\psi_{t}\right)=y_{t}$ and $D_{t} \psi_{t} \in \bar{H}\left(\psi_{t}\right)$. For $x \in F, \psi_{t}(x)$ is a curve in $M$ and 
$\left.D_{t} \psi_{t}(x)\right|_{t=0}$ is a tangent vector at $z=\psi(x)$. Let $H_{z}$ be the collection of all such vectors, then $H=\left\{H_{z} ; z \in M\right\}$ is a smooth distribution of transversal subspaces in $M . H$ is called a connection on the $(G, F)$-bundle $\pi: M \rightarrow N$. The curve $y_{t}$ is uniquely lifted to a curve $z_{t}$ in $M$ with $z_{0}=z$ such that $\pi\left(z_{t}\right)=y_{t}$ and $D_{t} z_{t} \in H\left(z_{t}\right)$. In fact, $z_{t}=\psi_{t}(x) . \psi_{t}$ will be called the horizontal lift in $G(M)$ and $z_{t}$ the horizontal lift in $M$ of $y_{t}$.

There is a function $h: G(M) \times F \rightarrow M$ such that $\psi(x)=h(\psi, x)$ for any $\psi \in G(M)$ and $x \in F$. Since

$$
H\left(z_{t}\right) d y_{t}=d z_{t}=d \psi_{t}(x)=D_{\psi} h\left(\psi_{t}, x\right) d \psi_{t}=D_{\psi} h\left(\psi_{t}, x\right) \bar{H}\left(\psi_{t}\right) d y_{t},
$$

we see immediately

$$
H_{z}=D_{\psi} h(\psi, x) \bar{H}_{\psi}
$$

for any $x \in F$ and $\psi \in G(M)$ with $z=\psi(x)$.

\section{INVARIANT OPERATORS}

In this section, we will assume that $\pi: M \rightarrow N$ is a $(G, F)$-bundle with a connection $H$. Let $A$ be a semielliptic operator on $F$. For any $g \in G, g(A)$ defines a semielliptic operator on $F$ by

$$
(g(A) f)(x)=(A(f \circ g))\left(g^{-1}(x)\right)
$$

for any function $f$ on $F$ and $x \in F . A$ is said to be $G$-invariant if $g(A)=A$ for any $g \in G$. For example, if $F$ has a Riemannian metric and $G$ is the group of isometries, then the Laplacian operator (Laplace-Betrami operator) on $F$ is $G$-invariant.

Assume that $A$ is $G$-invariant. We can define a semielliptic operator $A^{*}$ on $M$ as follows. For any function $f$ on $M$ and $z \in M$, choose $x \in F$ and $\psi \in G(M)$ such that $z=\psi(x)$. Let

$$
A^{*} f(z)=A(f \circ \psi)(x) .
$$

This definition of $A^{*}$ is independent of the choice of $x \in F$ and $\psi \in G(M)$ due to the $G$-invariance of $A, A^{*}$ is a vertical operator in the sense that $A^{*}(f \circ \pi)=0$ for any function $f$ on $N . A^{*}$ will be called the vertical operator induced by $A$.

Proposition 1. $A^{*}(H U)=(H U) A^{*}$ for any vector field $U$ on $N$. In particular, $A^{*}$ commutes with $\widetilde{B}$ for any semielliptic operator $B$ on $N$.

Proof. Fix $z \in M$ with $y=\pi(z)$. Choose $x \in F$ and $\psi \in G(M)$ with $\psi(x)=$ $z$. Let $y_{t}$ be the integral curve of $U$ with $y_{0}=y$ and $\psi_{t}$ be its horizontal lift in $G(M)$. Then $\psi_{t}(x)$ is the integral curve of $H U$ with $\psi_{0}(x)=\psi(x)=z$.

$$
A\left(f \circ \psi_{t}\right)(x)=A^{*} f\left(\psi_{t}(x)\right)
$$

for any function $f$ on $M$. Differentiate with respect to $t$, and we obtain

$$
\left.D_{t} A\left(f \circ \psi_{t}\right)(x)\right|_{t=0}=A[((H U) f) \circ \psi](x)=A^{*}(H U) f(z)
$$


and

$$
\left.D_{t} A^{*} f\left(\psi_{t}(x)\right)\right|_{t=0}=(H U) A^{*} f(\psi(x))=(H U) A^{*} f(z) .
$$

The conclusion follows. Q.E.D.

Proposition 2. $A^{*}+\widetilde{B}$ is a nondegenerate if and only if both $A$ and $B$ are nondegenerate.

Proof. Under local coordinates $x^{1}, x^{2}, \ldots, x^{k}$ on $F$ and $y^{1}, y^{2}, \ldots, y^{n}$ on $N, B$ is expressed by (2) and $A$ has the form

$$
A=(1 / 2) a^{\alpha \beta}(x)\left(\partial / \partial x^{\alpha}\right)\left(\partial / \partial x^{\beta}\right)+\text { first order derivatives. }
$$

Via the local trivialization map $\phi: 0 \times F \rightarrow \pi^{-1}(0), x^{1}, \ldots, x^{k}, y^{1}, \ldots, y^{n}$ become local coordinates on $M$. Under them, $A^{*}$ has the same form as $A$. Let

$$
H_{z}\left(\partial / \partial y^{j}\right)=\left(\partial / \partial y^{j}\right)+h_{j}^{\alpha}(z)\left(\partial / \partial x^{\alpha}\right)
$$

for any $z \in M$. Then

$$
\begin{aligned}
\widetilde{B}= & (1 / 2) g^{j k} h_{j}^{\alpha} h_{k}^{\beta}\left(\partial / \partial x^{\alpha}\right)\left(\partial / \partial x^{\alpha}\right)+g^{j 1} h_{1}^{\alpha}\left(\partial / \partial x^{\alpha}\right)\left(\alpha / \partial y^{j}\right) \\
& +(1 / 2) g^{j k}\left(\partial / \partial y^{j}\right)\left(\partial / \partial y^{k}\right)+\text { first order derivatives. }
\end{aligned}
$$

Let $Q$ be the matrix formed by the coefficients of the second order derivatives of $A^{*}+\widetilde{B}$ and let

$$
p=\left(\xi_{1}, \ldots, \xi_{k}, \eta_{1}, \ldots, \eta_{n}\right) \in R^{n+k} .
$$

Then

$$
p Q p^{*}=g^{j k}\left[\eta_{j}+h_{j}^{\alpha} \xi_{\alpha}\right]\left[\eta_{k}+h_{k}^{\beta} \xi_{\beta}\right]+a^{\alpha \beta} \xi_{\alpha} \xi_{\beta} .
$$

From this, it is easy to conclude that $Q$ is strictly positive definite if and only if $g^{j k}$ and $a^{\alpha \beta}$ are strictly positive definite. Q.E.D.

\section{RIEMANNIAN SUBMERSIONS}

Riemannian submersions provide important examples of fibre bundles. Assume that $M$ and $N$ are Riemannian manifolds and $M$ is complete. An onto map $\pi: M \rightarrow N$ is said to be a Riemannian submersion if for any $z \in M$ with $y=\pi(z), D \pi: H_{z} \rightarrow T_{y} N$ is an isometry, where $H_{z}$ is the subspace of $T_{z} M$ consisting of all vectors orthogonal to the fibre $F_{y}=\pi^{-1}(y)$ and is called the horizontal subspace at $z$. A curve in $M$ is said to be horizontal if it is tangent to horizontal subspaces. Any geodesic $y_{t}$ in $N$ lifts to horizontal geodesics starting from the fibre over $y_{0}$. This induces a diffeomorphism between fibres over $y_{0}$ and $y_{1}$. Hence, all fibres are diffeomorphic and $\pi: M \rightarrow N$ is a fibre bundle. Now assume that fibres are totally geodesic. Then the above diffeomorphism is isometric. Let $F$ be a Riemannian manifold isometric to some fibre and let $G$ be the group of isometries on $F$. Then $\pi: M \rightarrow N$ is a $(G, F)$ bundle and $H=\left\{H_{z} ; z \in M\right\}$ is a connection. See [H]. A trivial example of Riemannian submersion with totally geodesic fibres is the product Riemannian manifold $M=N \times F$ with $\pi: N \times F \rightarrow N$ being the projection. There are many interesting nontrivial examples, see [BB]. 
Proposition 3. Assume that $\pi: M \rightarrow N$ is a Riemannian submersion with totally geodesic fibres. Equip $M$ with the above $(G, F)$-bundle structure and connection. Let $A$ and $B$ be, respectively, the Laplacians on $F$ and $N$. Then $A^{*}+\widetilde{B}$ is the Laplacian on $M$.

Proof. Let $L$ be the Laplacian on $M$. Choose orthonormal vector fields $U_{1}, U_{2}, \ldots, U_{n}$ on $N$ and let $W_{1}, W_{2}, \ldots, W_{n}$ be their horizontal lifts. Choose orthonormal vector fields $V_{1}, V_{2}, \ldots, V_{k}$ on $M$ such that they span $T_{z} F_{\pi(z)}$ for any $z \in M$. Then

$$
L=V_{\alpha} V_{\alpha}-\nabla_{V_{\alpha}}^{\prime} V_{\alpha}+W_{j} W_{j}-\nabla_{W_{j}}^{\prime} W_{j}
$$

and

$$
B=U_{j} U_{j}-\nabla_{U_{j}} U_{j},
$$

where $\nabla$ and $\nabla^{\prime}$ are, respectively, the covariant differentiations on $N$ and $M$. Since each fibre is totally geodesic, the covariant differentiation on $F_{y}$ coincides with $\nabla^{\prime}$ and since each $\psi \in G(M)$ is an isometry, we have

$$
A^{*}=V_{\alpha} V_{\alpha}-\nabla_{V_{\alpha}}^{\prime} V_{\alpha} .
$$

Now the conclusion follows from the fact that if $W$ is a horizontal vector field on $M$, then $\nabla_{W} W$ is also horizontal, see Lemma 2 to [O]. Q.E.D.

Remark 2. Let $L$ and $L^{y}$ be, respectively, the Laplacians on $M$ and $F_{y}$. For any function $f$ on $M$, let $f_{y}$ be the restriction of $f$ to $F_{y}$. Define

$$
L^{v} f(z)=\left(L^{\pi(z)} f_{\pi(z)}\right)(z) .
$$

$L^{v}$ is called the vertical Laplacian and $L^{h}=L-L^{v}$ is called the horizontal Laplacian. The proof of Proposition 3 shows that $L^{v}=A^{*}$ and $L^{h}=\widetilde{B}$. By Proposition 1, $L^{v}$ ad $L^{h}$ commute, see [BB]. In fact, $L^{h}=\widetilde{B}$ holds if and only if the fibres are minimal.

Let $B$ be an elliptic operator on $N$ with local expression (2). Let $g_{j k}$ be the inverse matrix of $g^{j k}$. Define a Riemannian metric $($,$) on N$ by

$$
\left(\partial / \partial y^{j}, \partial / \partial y^{k}\right)=g_{j k} \text {. }
$$

This metric is called the natural metric of $B . B$ will be called a pre-Laplacian if it is the Laplacian with respect to its natural metric.

Proposition 4. Assume that $\pi: M \rightarrow N$ is a $(G, F)$-bundle with a connection $H, A$ is a $G$-invariant semielliptic operator on $F$ and $B$ is a semielliptic operator on $N$. Then $A^{*}+\widetilde{B}$ is a pre-Laplacian if and only if both $A$ and $B$ are pre-Laplacians.

Proof. Assume that $A$ and $B$ are the Laplacians with respect to their natural metrics. By [V], there is a unique way to define a Riemannian metric on $M$ such that $\pi: M \rightarrow N$ is a Riemannian submersion with totally geodesic fibres and $H$ is the distribution of horizontal subspaces. By Proposition $3, A^{*}+\widetilde{B}$ 
is the Laplacian on $M$. Now assume that $A^{*}+\widetilde{B}$ is a pre-Laplacian. By Proposition 2, both $A$ and $B$ are nondegenerate. Hence,

$$
A=A^{\prime}+V \text { and } B=B^{\prime}+U,
$$

where $A^{\prime}$ and $B^{\prime}$ are pre-Laplacians, $V$ and $U$ are vector fields. Then

$$
A^{*}+\widetilde{B}=\left(A^{\prime *}+\widetilde{B}^{\prime}\right)+\left(V^{*}+\widetilde{U}\right) .
$$

Since $\left(A^{\prime *}+\widetilde{B}^{\prime}\right)$ is a pre-Laplacian on $M$ and $V^{*}+\widetilde{U}=0$ if and only if both $V$ and $U$ vanish, we see that $V$ and $U$ must be zero. Q.E.D.

\section{DIFFUSIONS ON FIBRE BUNDLES}

Let $B$ be a semielliptic operator on some manifold $N$. A diffusion $Y_{t}$ with generator $B$ and initial condition $Y_{0}=y \in N$ is a stochastic process with a probability law $P$ such that $P\left(Y_{0}=y\right)=1$ and

$$
f\left(Y_{t}\right)-f\left(Y_{0}\right)-\int_{0}^{t} B f\left(Y_{s}\right) d s
$$

is a martingale for any function $f$ with compact support on $N$.

Assume that, locally, $B$ is expressed by (1). $Y_{t}$ is obtained as the solution to the following stochastic equation:

$$
d Y_{t}=U_{i}\left(Y_{t}\right) \circ d B_{t}^{i}+U^{\prime}\left(Y_{t}\right) d t,
$$

where $B_{t}=\left(B_{t}^{1}, B_{2}^{2}, \ldots, B_{t}^{s}\right)$ is a standard $s$-dimensional Brownian motion and $\circ d B_{t}^{i}$ denotes the Stratonovich differential. See [IW]. For the global existence of $Y_{t}$, see Chapter 2 in [A]. $Y_{t}$ will be called a $B$-diffusion. In this paper, any diffusion is a $B$-diffusion for some semielliptic operator $B$.

If $N$ is compact, $Y_{t} \in N$ for all $t<\infty$. In general, there is a positive random variable $\zeta$ which may take value $\infty$ such that $Y_{t} \in N$ for $t<\zeta$ and when $\zeta<\infty, Y_{t} \rightarrow \Delta$ as $t$ increases to $\zeta$, where $\Delta$ is the "point at infinity" used in the one point compactification of $N$. $\zeta$ is called the life time of $Y_{t}$.

Assume that $\pi: M \rightarrow N$ is a fibre bundle with standard fibre $F$ and a smooth distribution $H$ of transversal subspaces on $M$. Fix $y \in N$ and let $z \in F_{y}$. Define a diffusion $\widetilde{Y}_{t}$ on $M$ with initial condition $\widetilde{Y}_{0}=z$ by solving the following stochastic differential equation:

$$
d \widetilde{Y}_{t}=H\left(\tilde{Y}_{t}\right) \circ d Y_{t} .
$$

The generator of $\tilde{Y}_{t}$ is $\widetilde{B}$, the horizontal lift of $B$. Since $Y_{t}=\pi\left(\tilde{Y}_{t}\right)$, the life time of $\tilde{Y}_{t}$ is not greater than that of $Y_{t} . \tilde{Y}_{t}$ is called the horizontal lift of $Y_{t}$ with respect to $H$.

Now assume that $\pi: M \rightarrow N$ is a $(G, F)$-bundle and $H$ is a connection. Let $\psi \in G(M)$ with $y=\pi(\psi)$. We can define a diffusion $\Psi$, on $G(M)$ with 
$\Psi_{0}=\psi$ by solving the following stochastic differential equation:

$$
d \Psi_{t}=\bar{H}\left(\Psi_{t}\right) \circ d Y_{t}
$$

$\Psi_{t}$ is called the horizontal lift of $Y_{t}$ in $G(M)$. We have $\widetilde{Y}_{t}=\Psi_{t}(z)$.

Remark 3. On a $(G, F)$-bundle, the life times of $\tilde{Y}_{t}$ and $\Psi_{t}$ are in fact equal to that of $Y_{t}$. See [E, p. 174],

\section{COMPOSITION OF INDEPENDENT DIFFUSIONS}

In this section we will assume that $\pi: M \rightarrow N$ is a $(G, F)$-bundle with a connection $H$. Let $A$ be a semielliptic operator on $F, B$ be a semielliptic operator on $N, X_{t}$ be an $A$-diffusion with $X_{0}=x_{0}, Y_{t}$ be a $B$-diffusion with $Y_{0}=y_{0}$ and $\Psi_{t}$ be the horizontal lift in $G(M)$ of $Y_{t}$ with $\Psi_{0}=\psi_{0}$.

Proposition 5. Assume that $A$ is G-invariant and $X_{t}$ and $Y_{t}$ are independent. Then $Z_{t}=\Psi_{t}\left(X_{t}\right)$ is a diffusion on $M$ with generator $A^{*}+\widetilde{B}$.

Proof. Assume that $B$ and $Y_{t}$ satisfy (1) and (6), and $A$ and $X_{t}$ satisfy

$$
A=(1 / 2) V_{\alpha} V_{\alpha}+V^{\prime}
$$

and

$$
d X_{t}=V_{\alpha}\left(X_{t}\right) \circ d W_{t}^{\alpha}+V^{\prime}\left(X_{t}\right) d t,
$$

where $V_{1}, V_{2}, \ldots, V_{r}$ and $V^{\prime}$ are smooth vector fields on $F$ and

$$
W_{t}=\left(W_{t}^{1}, W_{t}^{2}, \ldots, W_{t}^{r}\right)
$$

is an $r$-dimensional standard Brownian motion which is independent with $B_{t}$. By (5), $A^{*}$ has the following expression at $z \in M$ :

$$
\begin{aligned}
A^{*}= & (1 / 2)\left[D \psi\left(V_{\alpha}\right)\right]\left[D \psi\left(V_{\alpha}\right)\right]+D \psi\left(V^{\prime}\right) \\
= & (1 / 2)\left[D_{x} h(\psi, x) V_{\alpha}(x)\right]\left[D_{x} h(\psi, x) V_{\alpha}(x)\right] \\
& +\left[D_{x} h(\psi, x) V^{\prime}(x)\right],
\end{aligned}
$$

where $x \in F$ and $\psi \in G(M)$ are chosen so that $z=\psi(x)$.

Since $Z_{t}=\Psi_{t}\left(X_{t}\right)=h\left(\Psi_{t}, X_{t}\right)$,

$$
\begin{aligned}
d Z_{t} & =D_{\psi} h\left(\Psi_{t}, X_{t}\right) \circ d \Psi_{t}+D_{x} h\left(\Psi_{t}, X_{t}\right) \circ d X_{t} \\
& =D_{\psi} h\left(\Psi_{t}, X_{t}\right) \bar{H}\left(\Psi_{t}\right) \circ d Y_{t}+D_{x} h\left(\Psi_{t}, X_{t}\right) \circ d X_{t} \\
& =H\left(Z_{t}\right) \circ d Y_{t}+D_{x} h\left(\Psi_{t}, X_{t}\right) \circ d X_{t} .
\end{aligned}
$$


For any function $f$ with compact support on $M$, we have, by Ito's formula,

$$
\begin{aligned}
f\left(Z_{t}\right) & -f\left(Z_{0}\right)=\int_{0}^{t} D f\left(Z_{u}\right) \circ d Z_{u} \\
= & \int_{0}^{t} D f\left(Z_{u}\right) H\left(Z_{u}\right) \circ d Y_{u}+\int_{0}^{t} D f\left(Z_{u}\right) D_{x} h\left(\Psi_{u}, X_{u}\right) \circ d X_{u} \\
= & \int_{0}^{t} D f\left(Z_{u}\right) H\left(Z_{u}\right) U_{i}\left(\pi\left(Z_{u}\right)\right) \circ d B_{u}^{i}+\int_{0}^{t} D f\left(Z_{u}\right) H\left(Z_{u}\right) U^{\prime}\left(\pi\left(Z_{u}\right)\right) d u \\
& +\int_{0}^{t} D f\left(Z_{u}\right) D_{x} h\left(\Psi_{u}, X_{u}\right) V_{\alpha}\left(X_{u}\right) \circ d W_{u}^{\alpha} \\
& +\int_{0}^{t} D f\left(Z_{u}\right) D_{x} h\left(\Psi_{u}, X_{u}\right) V^{\prime}\left(X_{u}\right) d u \\
= & \left.\int_{0}^{t}\left[H(z) U_{i}(\pi(z))\right] f\right|_{z=Z_{u}} \circ d B_{u}^{i}+\left.\int_{0}^{t}\left[H(z) U^{\prime}(\pi(z))\right] f\right|_{z=Z_{u}} d u \\
& +\left.\int_{0}^{t}\left[D_{x} h\left(\Psi_{u}, x\right) V_{\alpha}(x)\right] f\right|_{x=X_{u}} \circ d W_{u}^{\alpha}+\left.\int_{0}^{t}\left[D_{x} h\left(\Psi_{u}, x\right) V^{\prime}(x)\right] f\right|_{x=X_{u}} d u \\
= & \left.\int_{0}^{t}\left[H(z) U_{i}(\pi(z))\right] f\right|_{z=Z_{u}} d B_{u}^{i}+\left.\int_{0}^{t}\left[D_{x} h\left(\Psi_{u}, x\right) V_{\alpha}(x)\right] f\right|_{x=X_{u}} d W_{u}^{\alpha} \\
& +\left.\int_{0}^{t}\left\{(1 / 2)\left[H(z) U_{i}(\pi(z))\right]\left[H(z) U_{i}(\pi(z))\right] f+H(z) U^{\prime}(\pi(z)) f\right\}\right|_{z=Z_{u}} d u \\
& +\int_{0}^{t}\left\{(1 / 2)\left[D_{x} h\left(\Psi_{u}, x\right) V_{\alpha}(x)\right]\left[D_{x} h\left(\Psi_{u}, x\right) V_{\alpha}(x)\right] f\right. \\
= & \left.\left.{\operatorname{martingale}+\int_{0}^{t} \widetilde{B} f\left(Z_{u}\right) d u+\int_{0}^{t} A^{*} f\left(Z_{u}\right) d u .} d u\left(\Psi_{u}, x\right) V^{\prime}(x)\right] f\right\}\left.\right|_{x=X_{u}} d u
\end{aligned}
$$

This shows that $Z_{t}$ is a diffusion with generator $A^{*}+\widetilde{B}$. The above computation is justified by the fact that $B$. and $W$. are independent, so when we integrate with respect to $\circ d B_{u}, X$. can be regarded as a fixed path and when we integrate with respect to $\circ d W_{u}, Y$. can be regarded as a fixed path. Q.E.D. Remark 4. The life time of $\Psi_{t}\left(X_{t}\right)$ is the smaller of the life times of $X_{t}$ and $Y_{t}$.

Remark 5. Let $Z_{t}$ be a diffusion on $M$. If $Z_{t}$ can be factored through two independent diffusions $X_{t}$ on $F$ and $Y_{t}$ on $N$ as in Proposition 5, then $\pi\left(Z_{t}\right)=Y_{t}$. In general, $\pi\left(Z_{t}\right)$ is not a diffusion on $N$. We would like to have a simple condition for this to happen.

A semielliptic operator $L$ on $M$ is said to be projectable if there is a differential operator $B$ (necessarily semielliptic) on $N$ such that

$$
L(f \circ \pi)=(B f) \circ \pi
$$

for any function $f$ on $N$. We will say that $L$ projects to $B$. It is clear that if $L=A^{*}+\widetilde{B}$, then $L$ projects to $B$. We have the following simple result. 
If $L$ projects to $B$, then $\pi\left(Z_{t}\right)$ is a $B$-diffusion for any $L$-diffusion $Z_{t}$. Conversely, if this is true, then $L$ projects to $B$.

To show this, let $Y_{t}=\pi\left(Z_{t}\right)$. If $L$ projects to $B$, then

$$
f\left(Y_{t}\right)-f\left(Y_{0}\right)-\int_{0}^{t} B f\left(Y_{u}\right) d u=f \circ \pi\left(Z_{t}\right)-f \circ \pi\left(Z_{t}\right)-\int_{0}^{t} L(f \circ \pi)\left(Z_{u}\right) d u
$$

is a martingale, so $Y_{t}$ is a $B$-diffusion. Conversely, if the left-hand side of the above expression is a martingale, then

$$
\int_{0}^{t} B f\left(Y_{u}\right) d u-\int_{0}^{t} L(f \circ \pi)\left(Z_{u}\right) d u
$$

is a martingale. This implies that it is actually zero and (9) holds.

Let $N$ be a Riemannian manifold and $B$ be its Laplacian. A $B$-diffusion is said to be a Brownian motion on $N$. Assume that $\pi: M \rightarrow N$ is a Riemannian submersion with minimal fibres. Then the Laplacian on $M$ projects to the Laplacian on $N$, see [W]. We immediately obtain Elworthy's result [E] that if $Z_{t}$ is a Brownian motion on $M$, then $\pi\left(Z_{t}\right)$ is a Brownian motion on $N$ provided that fibres are minimal.

Proposition 6. Let $\pi: M \rightarrow N$ be a $(G, F)$-bundle with a connection, $Z_{t}$ be an $L$-diffusion on $M$ and $Y_{t}=\pi\left(Z_{t}\right)$. Assume that $L=A^{*}+\widetilde{B}$ for a $G$-invariant semielliptic operator $A$ on $F$ and a semielliptic operator $B$ on $N$. Then $Y_{t}$ is a B-diffusion on $N$ and $X_{t}=\Psi_{t}^{-1}\left(Z_{t}\right)$ is an A-diffusion on $F$ which is independent of $Y_{t}$, where $\Psi_{t}$ is a horizontal lift in $G(M)$ of $Y_{t}$. In particular, $Z_{t}=\Psi_{t}\left(X_{t}\right)$.

Proof. By Proposition 5, if we choose an $A$-diffusion $X_{t}^{\prime}$ with the same initial condition as $X_{t}$ such that $X^{\prime}$ and $Y$ are independent, then $Z_{t}^{\prime}=\Psi_{t}\left(X_{t}^{\prime}\right)$ is a diffusion on $M$ which has the same probability law as $Z_{t}$ and $Y_{t}=\pi\left(Z_{t}^{\prime}\right)$. From this it follows that the joint law of $X^{\prime}$ and $Y$ is the same as that of $X$ and $Y$, so $X$ and $Y$ are independent. Q.E.D.

The factorization of Elworthy and Kendall follows from Propositions 3 and 6.

Corollary 1. Let $\pi: M \rightarrow N$ be a Riemannian submersion with totally geodesic fibres and $Z_{t}$ be a Brownian motion on $M$. Equip $M$ with the natural $(G, F)$ bundle structure (see $\S 4)$. Then $Y_{t}=\pi\left(Z_{t}\right)$ is a Brownian motion on $N$ and $X_{t}=\Psi_{t}^{-1}\left(Z_{t}\right)$ is a Brownian motion on $F$, where $\Psi_{t}$ is a horizontal lift in $G(M)$ of $Y_{t}$. Moreover, $X$ and $Y$ are independent.

Remark 6. Let $Y_{t}$ be a $B$-diffusion on $N . Y_{t}$ is said to be nondegenerate if $B$ is nondegenerate and is said to be a pre-Brownian motion if $B$ is a pre-Laplacian. By Propositions 2 and 4, we have the following interesting observation. Let $\pi: M \rightarrow N$ be a $(G, F)$-bundle with a connection, $X_{t}$ be a diffusion on $F$ with $G$-invariant generator, $Y_{t}$ be a diffusion on $N, \Psi_{t}$ be a horizontal lift in $G(M)$ of $Y_{t}$ and $Z_{t}=\Psi_{t}\left(X_{t}\right)$. Then $Z_{t}$ is nondegenerate if 
and only if both $X_{t}$ and $Y_{t}$ are nondegenerate, $Z_{t}$ is a pre-Brownian motion if and only if both $X_{t}$ and $Y_{t}$ are pre-Brownian motions.

\section{STOCHASTIC FLOWS OF DIFFEOMORPHISMS}

Let $\pi: M \rightarrow N$ be a fibre bundle with a distribution $H$ of transversal subspaces on $M$. Fix $y \in N$ and $z \in F_{y}$, let $Y_{t}$ be a diffusion on $N$ with $Y_{0}=y$ and $\tilde{Y}_{t}$ be its horizontal lift in $M$ with $\tilde{Y}_{0}=z$. We will denote $\tilde{Y}_{t}$ by $\Phi_{t}(z)$. Almost surely, for fixed $t>0, \Phi_{t}$ is a map from $F_{y}$ into the fibre over $Y_{t}$ with the understanding that possibly for some $z^{\prime} \in F_{y}, \Phi_{t}\left(z^{\prime}\right)=\Delta$, due to the fact that the life time of $\Phi$. $\left(z^{\prime}\right)$ may be smaller than $t$. If $\pi: M \rightarrow N$ is a $(G, F)$-bundle and $H$ is a connection, then, by Remark 3, almost surely, $\Phi_{t}$ is either a diffeomorphism from $F_{y}$ onto the fibre over $Y_{t}$ or the map $F_{y} \rightarrow\{\Delta\}$, depending on whether $t$ is smaller than the life time of $Y$.

Let $R$ be a vertical semielliptic operator on $M$, i.e. $R(f \circ \pi)=0$ for any function $f$ on $N$. We can define a semielliptic operator $R^{y}$ on $F_{y}$ as follows. For any function $h$ on $F_{y}$, extend it to be a function $f$ on $M$ such that $f=h$ on $F_{y}$ and define $R^{y} h(z)=R f(z)$. This definition of $R^{y}$ is independent of the choice of $f$ due to the fact that if $f$ is constant on $F_{y}$, then $R f=0$ on $F_{y}$. This fact is checked easily by using local coordinates.

Let $X_{t}^{\prime}$ be an $R^{y}$-diffusion on $F_{y}$ with $X_{0}^{\prime}=z$. We would like to know whether $\Phi_{t}\left(X_{t}^{\prime}\right)$ is a diffusion on $M$. If $\pi: M \rightarrow N$ is a $(G, F)$-bundle, $H$ is a connection and $R=A^{*}$ for some $G$-invariant operator $A$ on $F$, then, by Proposition 5, $\Phi_{t}\left(X_{t}^{\prime}\right)$ is a diffusion. The purpose of this section is to show that, under some additional conditions, the converse of this statement also holds, i.e . if $\Phi_{t}\left(X_{t}^{\prime}\right)$ is a diffusion on $M$, then there is a $G$-structure on the fibre bundle under which, $H$ is a connection and $R=A^{*}$ for some $G$-invariant operator $A$ on $F$. We will first consider the case of a Riemannian submersion, then that of a fibre bundle with nondegenerate operators.

Proposition 7. Let $\pi: M \rightarrow N$ be a Riemannian submersion with complete $M$. Assume that for any $y \in N$ and $z \in F_{y}, \Phi_{t}\left(X_{t}^{\prime}\right)$ is a diffusion on $M$, where $X_{t}^{\prime}$ is a Brownian motion on $F_{y}$ with $X_{0}^{\prime}=z, Y_{t}$ is a Brownian motion on $N$ with $Y_{0}=y$ and $\Phi_{t}\left(z^{\prime}\right)$ is the horizontal lift in $M$ of $Y_{t}$ with $\Phi_{0}\left(z^{\prime}\right)=z^{\prime}$ for any $z^{\prime} \in F_{y}$. Then the fibres are totally geodesics. In particular, $\Phi_{t}\left(X_{t}^{\prime}\right)$ is a Brownian motion on $M$.

Proof. $\Phi_{t}$ can be considered as the stochastic flow of local diffeomorphisms associated with the stochastic differential equation

$$
d Z_{t}=H\left(Z_{t}\right) \circ d Y_{t}=\left(H U_{i}\right)\left(Z_{t}\right) \circ d B_{t}^{i}+\left(H U^{\prime}\right)\left(Z_{t}\right) d t .
$$

The results of $[\mathrm{K}]$ will be applied. 
Let $Z_{t}=\Phi_{t}\left(X_{t}^{\prime}\right)$ and let $L$ be its generator. By the generalized Ito formula, see [K, Chapter I, Theorem 8.3], we have

$$
f\left(Z_{t}\right)-f\left(Z_{0}\right)=\int_{0}^{t} D f\left(Z_{u}\right) H\left(Z_{u}\right) \circ d Y_{u}+\int_{0}^{t} D f\left(Z_{u}\right) D \Phi_{u}\left(X_{u}^{\prime}\right) \circ d X_{u}^{\prime}
$$

for any function $f$ with compact support on $M$. A computation similar to that used in the proof of Proposition 5 yields

$$
f\left(Z_{t}\right)-f\left(Z_{0}\right)=\text { martingale }+\int_{0}^{t} \widetilde{B} f\left(Z_{u}\right) d u+\int_{0}^{t} L^{y}\left(f \circ \Phi_{u}\right)\left(X_{u}^{\prime}\right) d u,
$$

where $B$ and $L^{y}$ are, respectively, the Laplacians on $N$ and $F_{y}$. On the other hand,

$$
f\left(Z_{t}\right)-f\left(Z_{0}\right)=\text { martingale }+\int_{0}^{t} L f\left(Z_{u}\right) d u .
$$

Putting $L^{\prime}=L-\widetilde{B}$, we obtain

$$
L^{y}\left(f \circ \Phi_{t}\right)\left(X_{t}^{\prime}\right)=L^{\prime} f\left(\Phi_{t}\left(X_{t}^{\prime}\right)\right) .
$$

Since $X_{t}^{\prime}$ is nondegenerate and $X_{t}^{\prime}$ and $\Phi_{t}$ are independent, this implies that for any $z^{\prime} \in F_{y}$,

$$
L^{y}\left(f \circ \Phi_{t}\right)\left(z^{\prime}\right)=L^{\prime} f\left(\Phi_{t}\left(z^{\prime}\right)\right) .
$$

Setting $t=0$, we see immediately that $L^{\prime}$ is just the vertical Laplacian on $M$ (cf. Remark 2). Replacing $f$ by $f \circ \Phi_{t}^{-1}$, we have

$$
L^{\prime}\left(f \circ \Phi_{t}^{-1}\right)\left(\Phi_{t}(z)\right)=L^{y} f(z)=L^{\prime} f(z) .
$$

By [K, Chapter III, 2.2], the following expression is identically zero:

$$
\int_{0}^{t}\left[H U_{i}, L^{\prime}\right]\left(f \circ \Phi_{u}^{-1}\right)\left(\Phi_{u}(z)\right) \circ d B_{u}^{i}+\int_{0}^{t}\left[H U^{\prime}, L^{\prime}\right]\left(f \circ \Phi_{u}^{-1}\right)\left(\Phi_{u}(z)\right) d u,
$$

where $\left[H U_{i}, L^{\prime}\right]=\left(H U_{i}\right) L^{\prime}-L^{\prime}\left(H U_{i}\right)$. In fact, in Theorem $2.2, L^{\prime}$ is assumed to be a vector field, but its proof applies to any semielliptic operator $L^{\prime}$. Since the above expression vanishes, we obtain

$$
\left[H U_{i}, L^{\prime}\right]=0 \text { and }\left[H U^{\prime}, L^{\prime}\right]=0 .
$$

$U_{1}, U_{2}, \ldots, U_{n}$ can be chosen to be any orthonormal vector fields on $N$. In particular, we may assume that they are parallel along any geodesic starting from $y$. Let $y_{t}$ be such a geodesic, then it is the integral curve of a vector field obtained as a linear combination of $U_{i}, i=1,2, \ldots, n$. For $z^{\prime} \in F_{y}$, let $\phi_{t}\left(z^{\prime}\right)$ be the horizontal lift in $M$ of $y_{t}$ with $\phi_{0}\left(z^{\prime}\right)=z^{\prime} . \phi_{t}\left(z^{\prime}\right)$ is a horizontal geodesic in $M$. It follows from the completeness of $M$ that $\phi_{t}\left(z^{\prime}\right)$ is defined for any $t$ where $y_{t}$ is defined. Since $\phi_{t}\left(z^{\prime}\right)$ is the integral curve of a linear combination of $H U_{i}, i=1,2, \ldots, n$, and $\left[H U_{i}, L^{\prime}\right]=0$, we have

$$
L^{\prime} f\left(\phi_{t}\left(z^{\prime}\right)\right)=L^{\prime}\left(f \circ \phi_{t}\right)\left(z^{\prime}\right) \text {. }
$$


Since $L^{\prime}$ is the vertical Laplacian, $\phi_{t}$ is in fact an isometry from $F_{y}$ onto the fibre over $y_{t}$. A variational argument in [V] shows that all fibres are totally geodesic. Q.E.D.

A distribution $H$ of transversal subspaces on $M$ is said to be complete if for any $y \in N, z \in F_{y}$ and any curve $y_{t}, t \in[0,1]$, in $N$ with $y_{0}=y$, there is a (unique) curve $z_{t}, t \in[0,1]$, in $M$ with $z_{0}=z$ such that $\pi\left(z_{t}\right)=y_{t}$ and $D_{t} z_{t} \in H\left(z_{t}\right)$. If $\pi: M \rightarrow N$ has a $G$-structure and $H$ is a connection, then $H$ is complete.

Proposition 8. Let $\pi: M \rightarrow N$ be a fibre bundle with standard fibre $F, H$ be a complete distribution of transversal subspaces on $M, R$ be a vertical semielliptic operator on $M$ such that for any $y \in N, R^{y}$ is nondegenerate and $B$ be an elliptic operator on $N$. If for any $y \in N$ and $z \in F_{y}, \Phi_{t}\left(X_{t}^{\prime}\right)$ is a diffusion on $M$, where $X_{t}^{\prime}$ is an $R^{y}$-diffusion on $F_{y}$ with $X_{0}^{\prime}=z, Y_{t}$ is a B-diffusion on $N$ with $Y_{0}=y$ and $\Phi_{t}\left(z^{\prime}\right)$ is the horizontal lift in $M$ of $Y_{t}$ with $\Phi_{0}\left(z^{\prime}\right)=z^{\prime}$ for any $z^{\prime} \in F_{y}$, then $\pi: M \rightarrow N$ has a structure group $G$ such that $H$ is a connection, $R=A^{*}$ for some G-invariant operator $A$ on $F$ and $\Phi_{t}\left(X_{t}^{\prime}\right)$ is a diffusion with generator $R+\widetilde{B}$. In fact, $\Phi_{t}\left(X_{t}^{\prime}\right)=\Psi_{t}\left(X_{t}\right)$ for some A-diffusion $X_{t}$ on $F$ and a horizontal lift $\Psi_{t}$ in $G(M)$ of $Y_{t}$.

Proof. As in the proof of Proposition 7, we can show that for any function $f$ with compact support on $M$ and any $z^{\prime} \in F_{y}$,

$$
R\left(f \circ \Phi_{t}\right)\left(z^{\prime}\right)=R f\left(\Phi_{t}\left(z^{\prime}\right)\right) \text { and }\left[H U_{i}, R\right]=0,
$$

where $U_{i}, i=1,2, \ldots, n$, are vector fields in (1). Let $y_{t}, t \in[0,1]$, be the integral curve of a linear combination of $U_{i}, i=1,2, \ldots, n$, with $y_{0}=y$. The completeness of $H$ ensures that the horizontal lift $\phi_{t}\left(z^{\prime}\right)$ in $M$ of $y_{t}$ exists for any $t \in[0,1]$ and $z^{\prime} \in F_{y}$. We have

$$
R\left(f \circ \phi_{t}\right)\left(z^{\prime}\right)=R f\left(\phi_{t}\left(z^{\prime}\right)\right) .
$$

$\phi_{t}$ is a diffeomorphism from $F_{y}$ onto the fibre over $y_{t}$.

For $\eta=\left(\eta^{1}, \eta^{2}, \ldots, \eta^{n}\right) \in R^{n}$ and $z^{\prime} \in F_{y}$, let $y_{t}^{\eta}$ be the integral curve of $\eta^{j} U_{j}$ with $y_{0}^{\eta}=y$ and let $\phi_{t}^{\eta}\left(z^{\prime}\right)$ be the horizontal lift in $M$ of $y_{t}^{\eta}$ with $\phi_{0}^{\eta}\left(z^{\prime}\right)=z^{\prime}$. For "small" $\eta$, both $y_{t}^{\eta}$ and $\phi_{t}^{\eta}$ are defined for $t \in[0,1]$ and $\phi_{1}^{\eta}$ is a diffeomorphism from $F_{y}$ onto the fibre over $y_{1}^{\eta}$. Since $B$ is nondegenerate, $U_{1}, U_{2}, \ldots, U_{n}$ are linearly independent at each point of $N$, so $\eta^{1}, \eta^{2}, \ldots, \eta^{n}$ form local coordinates around $y$.

Fix a diffeomorphism $\psi: F \rightarrow F_{y}$ and define $\phi(\eta, x)=\phi_{1}^{\eta}(\psi(x))$ for $x \in$ $F$. $\phi$ is a trivialization map $0 \times F \rightarrow \pi^{-1}(0)$ for some open neighborhood 0 of $y$. Let $A$ be the elliptic operator on $F$ defined by

$$
A h(x)=R\left(h \circ \psi^{-1}\right)(\psi(x))
$$


for any function $h$ on $F$ and let $G$ be the Lie group of diffeomorphisms $g: F \rightarrow F$ satisfying $g(A)=A$. Denote $\phi(\eta, \cdot)$ by $\phi^{\eta}$ and for any $y^{\prime} \in 0$ with coordinates $\eta=\left(\eta^{1}, \eta^{2}, \ldots, \eta^{n}\right)$, define $G_{y^{\prime}}=\left\{\phi^{\eta} \circ g ; g \in G\right\}$. Then $G_{y^{\prime}}, y^{\prime} \in 0$, form a $G$-structure on the restricted fibre bundle $\pi: \pi^{-1}(0) \rightarrow 0$. This $G$-structure extends to the whole bundle $\pi: M \rightarrow N$ and has the required properties. Q.E.D:

\section{APPENDIX}

If we redefine a semielliptic operator $B$ to be one which can be locally expressed by (2), then all our results will continue to hold under this slightly more general definition. The author wishes to thank the referee for this observation.

In fact, the definition of $A^{*}$ is not affected by this new definition of semielliptic operators. Since the definition of nondegenerate semielliptic operators remains the same, so do Propositions 3, 7, 8 and Corollary 1 . A careful inspection shows that the Propositions 2, 4 and 6 require no new proofs. Therefore, the only things which need to be modified are the definitions of $\widetilde{B}, \widetilde{Y}$ and $\Psi$, and the proofs of Propositions 1 and 5.

With our old definition of semielliptic operators, $\widetilde{B}$ is defined by (3). A direct computation using local coordinates $\left(x^{1}, \ldots, x^{k}, y^{1}, \ldots, y^{n}\right)$ yields

$$
\begin{aligned}
\widetilde{B}= & \frac{1}{2} g^{j k}\left[\partial_{j} \partial_{k}+2 h_{k}^{\alpha} \partial_{j} \partial_{\alpha}+h_{j}^{\alpha} h_{k}^{\beta} \partial_{\alpha} \partial_{\beta}\right]+b^{j} \partial_{j} \\
& +\left[\frac{1}{2} g^{j k}\left(\partial_{j} h_{k}^{\alpha}+h_{j}^{\beta} \partial_{\beta} h_{k}^{\alpha}\right)+b^{k} h_{k}^{\alpha}\right] \partial_{\alpha},
\end{aligned}
$$

where $\partial_{j}=\partial / \partial y^{j}, \partial_{k}=\partial / \partial y^{k}, \partial_{\alpha}=\partial / \partial x^{\alpha}$ and $\partial_{\beta}=\partial / \partial x^{\beta}$, and $h_{j}^{\alpha}$ are defined by $H\left(\partial_{j}\right)=\partial_{j}+h_{j}^{\alpha} \partial_{\alpha}$ for the fixed distribution $H$ of transversal subspaces on $M$. We can use (10) to define $\widetilde{B}$ and this definition is valid when $B$ is defined by (2).

The first part of Proposition $1, A^{*}(H U)=(H U) A^{*}$, is not affected by the change of the definition of semielliptic operators. The second part, the commutativity of $A^{*}$ and $\widetilde{B}$, which is now no longer a direct consequence of the first part, can be verified directly using (10).

Let $Y_{t}$ be a $B$-diffusion, where $B$ is defined by (2). By [IW, Chapter VI, $\S 6], Y_{t}$ is obtained as the solution of the following Ito equation:

$$
d Y_{t}^{j}=\sigma_{1}^{j}\left(Y_{t}\right) d B_{t}^{1}+b^{j}\left(Y_{t}\right) d t,
$$

where $\sigma_{1}^{j}$ satisfying $\sigma_{1}^{j} \sigma_{1}^{k}=g^{j k}$ can be chosen to be Lipschitz continuous. Now, (11) together with

$$
d X_{t}^{\alpha}=\left(\sigma_{1}^{j} h_{j}^{\alpha}\right)\left(X_{t}, Y_{t}\right) d B_{t}^{1}+\left[\frac{1}{2} g^{j k}\left(\partial_{j} h_{k}^{\alpha}+h_{j}^{\beta} \partial_{\beta} h_{k}^{\alpha}\right)+b^{j} h_{j}^{\alpha}\right]\left(X_{t}, Y_{t}\right) d t
$$

defines a $\widetilde{B}$-diffusion

$$
\tilde{Y}_{t}=\left(X_{t}^{1}, \ldots, X_{t}^{k}, Y_{t}^{1}, \ldots, Y_{t}^{n}\right)
$$

on $M$. This is the horizontal lift in $M$ of $Y_{t}$. 
The horizontal lift $\Psi_{t}$ in $G(M)$ of $Y_{t}$ can be defined similarly and we have $\tilde{Y}_{t}=\Psi_{t}(z)$ if $\tilde{Y}_{0}=z$.

Finally, Proposition 5 can be proved by a computation essentially similar to the old one but we have to use Ito's integrals instead of Stratonovich integrals.

\section{REFERENCES}

[A] R. Azencott, Diffusions sur les variétés. Généralités, Astérisque 84-85 (1981), 17-32.

[BB] L. Bergery and J. Bourguignon, Laplacians and Riemannian submersions with totally geodesic fibres, Illinois J. Math. 26 (1982), 181-200.

[E] K. D. Elworthy, Stochastic differential equations on manifolds, London Math. Soc. Lecture Note Ser., no. 70, Cambridge Univ. Press, 1982.

[EK] K. D. Elworthy and W. S. Kendall, Factorization of harmonic maps and Brownian motions, Local Time to Global Geometry, Control and Physics (K. D. Elworthy, ed.), Pitman Res. Notes in Math. Ser. 150, 1985, pp. 75-83.

[H] R. Hermann, A sufficient condition that a mapping of Riemannian manifolds be a fibre bundle, Proc. Amer. Math. Soc. 11 (1960), 236-242.

[IW] N. Ikeda and S. Watanabe, Stochastic differential equations and diffusion processes, NorthHolland, Amsterdam, 1981.

[K] H. Kunita, Stochastic differential equations and stochastic flows of diffeomorphisms, Lecture Notes in Math., vol. 1079, Springer-Verlag, Berlin and New York, pp. 143-303.

[KN] S. Kobayashi and K. Nomizu, Foundations of differential geometry. I, Interscience, New York, 1963.

[O] B. O'Neil, The fundamental equations of a submersion, Michigan Math. J. 13 (1966), 459-469.

[V] J. Vilms, Totally geodesic maps, J. Differential Geometry 4 (1970), 73-79.

[W] B. Watson, Manifold maps commuting with the Laplacian, J. Differential Geometry 8 (1973), 85-94.

Department of Mathematics, University of Florida, Gainesville, Florida 32611

Current address: Department of Mathematics, Nankai University, Tianjin, P.R. China 